\title{
Modelling the Interaction Forces between an Ideal Measurement Surface and the Stylus Tip of a Novel Vibrating Micro-scale CMM Probe
}

\author{
J.D. Claverley ${ }^{1}$, A. Georgi ${ }^{2}$, and R.K. Leach ${ }^{1}$ \\ ${ }^{1}$ National Physical Laboratory, Hampton Road, Teddington, UK, TW11 0LW \\ Tel.: +442089436242 \\ james.claverley@npl.co.uk \\ ${ }^{2}$ The University of North Carolina at Charlotte, NC, USA
}

\begin{abstract}
This paper describes the development of an analytical model to describe a novel three-axis vibrating micro-scale probe for micro-co-ordinate measuring machines (micro-CMMs). The micro-CMM probe is vibrated in three axes, in order to address the problems inherent with micro- and nano-scale co-ordinate measurements caused by surface interaction forces. These surface forces have been investigated and a mathematical model describing an ideal probing situation has been developed. The vibration amplitude required for the probe to overcome the surface interaction forces has been calculated using this model. The results of initial vibration experiments are reported and the suitability of the probe to counteract the surface interaction forces is confirmed.
\end{abstract}

Keywords: micro-CMM probe, surface interaction forces.

\section{Introduction}

As micro- and nano-scale engineering becomes ever more prevalent in many aspects of modern manufacturing, there is an urgent need for accurate and traceable microand nano-metrology. Recent advances in co-ordinate metrology, specifically the development of micro-CMMs, have enabled significant improvements to be made in three dimensional traceable micro- and nano-metrology. These micro-CMMs have highly advanced and rigid metrological frames and high accuracy measurement scales capable of nanometre resolution. The measuring accuracy of micro-CMMs has been investigated and found to be limited by the probe systems, rather than the machines themselves [1]. The National Physical Laboratory (NPL) is continuing to work on advanced micro-CMM probe design with the aim of improving the overall accuracy of modern micro-CMMs to less than $100 \mathrm{~nm}$ [2].

Simply reducing the size of current CMM probes is no longer a viable method of producing an accurate micro-CMM probe. Such reduction in size has been taken to its limit by various research projects both within NPL [2] and at other laboratories around the world [3]. Any work done to continue the advancement of micro-CMM probe research should implement a total redesign to take into account the need for low contact probing forces and the effect of the surface interaction forces. Research that has addressed some of these issues includes the development of a high aspect ratio 
fibre-based probe for 2D co-ordinate metrology, low force silicon based microprobes with piezoresistive strain gauges, CMM probes that optically measure probe deflection and ultra low force scanning probes that use laser trapped micro spheres (see reference [3] for a review of current probing technology).

To address the effects of surface forces and the need for low force probing, a vibrating micro-CMM probe has been designed and produced. It consists of a triskelion (three legged) flexure device and a micro-stylus [4]. The vibration of the probe will be controlled so that the stylus tip is always vibrating normal to the measurement surface and so that the acceleration of the stylus tip is sufficient to counteract the surface interaction forces. The vibrating aspect of this probe enables it to work in a noncontact fashion that will, theoretically, reduce the probing force incident on the measurement surface to zero.

The micro-CMM probe is made to vibrate by using six piezoelectric (PZT) thin film actuators (two on each flexure) and two PZT sensors on either end of each flexure which detect any change in vibration amplitude. The size of the flexures, the size, shape, and sensitivity of the sensors, and the ability of the triskelion device to exert forces isotropically on the measurement surface, have been subject to extensive research. The basic design of the new vibrating micro-CMM probe is shown in Fig. 1.

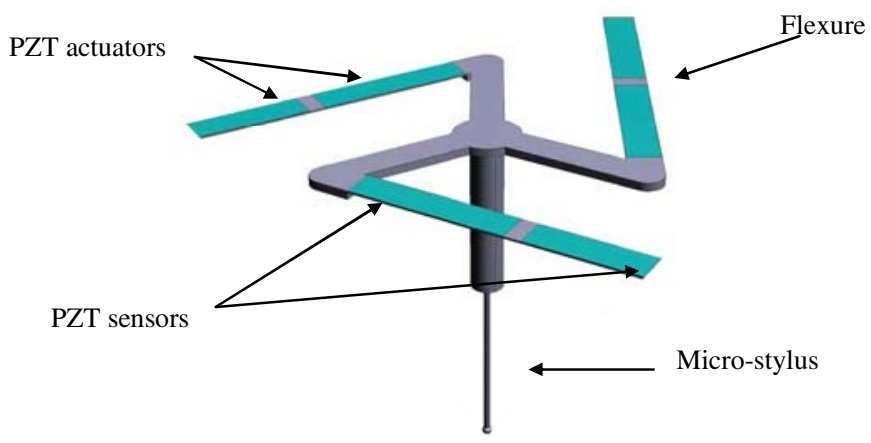

Fig. 1. A schematic diagram of the vibrating micro-CMM probe

\section{Surface Force Theory}

An analytical model was created to describe the effects that the surface forces will have on the oscillatory behaviour of the probe and to determine whether the amplitude of the probe was sufficient to counteract the surface interaction forces.

It is assumed that the device can be directly compared to a driven oscillator subject to a damping force. The second order differential equation that describes this situation is

$$
m \frac{\mathrm{d}^{2} z}{\mathrm{~d} t^{2}}+b \frac{\mathrm{d} z}{\mathrm{~d} t}+k z=F_{0} \cos (\omega t)
$$


where $m$ is the mass of the oscillator, $z$ is the position of the bottom of the probe tip, $b$ is the damping coefficient, $k$ is the spring constant of the oscillator, $F_{0}$ is the driving force and $\omega$ is the angular frequency. The spring constant of the probe has been found to be $15 \mathrm{~N} \cdot \mathrm{m}^{-1}$ using a finite element model.

A good understanding of the oscillatory behaviour of the probe can be gained from equation 1. To solve for $z$, an appreciation of $b$, and hence the surface forces, is required. A set of models was used to represent the surface interaction forces and a more complete model of the changing frequency and amplitude of the probe tip was calculated using equation 1 . Note that a similar investigation has been conducted for a high accuracy vibrating probe being developed by the University of North Carolina at Charlotte [5].

\subsection{The Capillary Force}

Capillary forces play a dominant role in micro- and nano-scale interactions [6]. The basis of the capillary force is the thin liquid film layers that accumulate on surfaces from condensation and contamination. As two surfaces are brought together the films join and the surface tension causes an adhesion effect. At the micro- and nano-scale these forces can often be the dominant force governing interactions. Contact angle plays a large role in determining the magnitude of capillary forces and can be affected by the material surface chemistry as well as the surface form and texture. A schematic representation of contact between a sphere and a surface with a liquid layer is shown in Fig. 2.

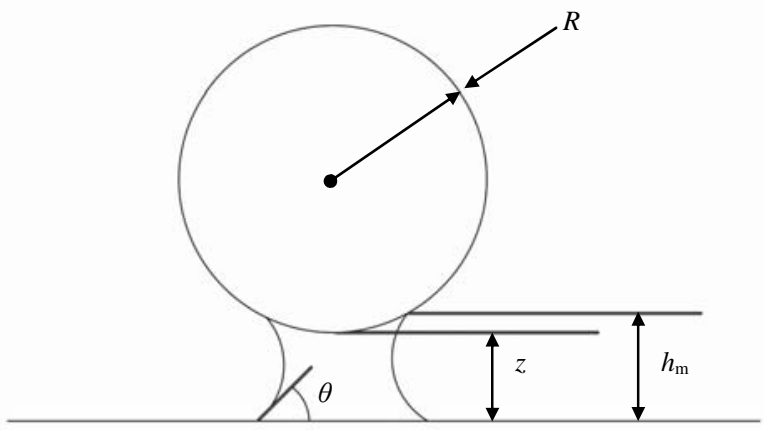

Fig. 2. A schematic representation of a sphere, radius $R$, interacting with a surface with a liquid layer. The diagram shows the variables $z$ (distance from surface), $h_{\mathrm{m}}$ (height of meniscus) and $\theta$ (the contact angle).

The equation used to describe the capillary force between a sphere and a plane surface is

$$
F_{1}=\frac{4 \pi R \gamma_{\mathrm{L}} \cos \theta}{1+\frac{z}{h_{\mathrm{m}}-z}}
$$

where $\gamma_{L}$ is the surface tension of the liquid [6]. 


\subsection{The Electrostatic Force}

Electrostatic forces result from electrical charge generation or charge transfer between two surfaces. Under most circumstances the amount of charge separated is small due to the breakdown strength of the surrounding air. However, as gap size decreases below the mean free path for air (approximately $1 \mu \mathrm{m}$ ), the magnitude of the charge density can increase by orders of magnitude [7]. The strength of the electrostatic force also depends strongly on the material characteristics of the interacting bodies. The electrostatic force is determined by the electrical potential difference of the two interacting surfaces and is given by

$$
F_{2}=\frac{\varepsilon_{0} U^{2} \pi R^{2}}{z^{2}}
$$

where $\varepsilon_{0}$ is the permittivity of free space and $U$ is the potential difference between the two interacting surfaces [8].

\subsection{The Van der Waals Force}

Van der Waals forces arise from the polarisation of atoms and molecules as they are drawn together. A schematic representation of contact between a sphere and a surface is shown in Fig. 3.

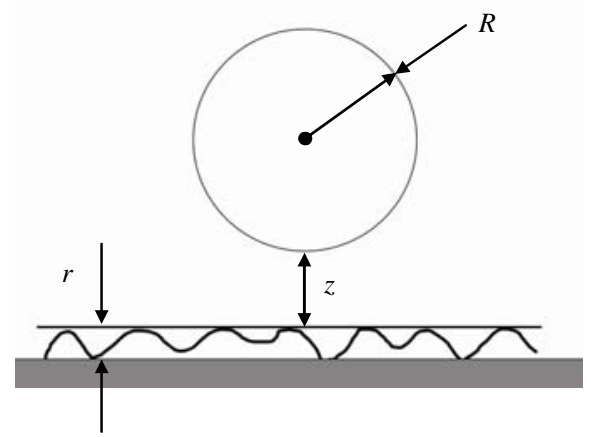

Fig. 3. Reduced Van der Waals forces depend on a surface parameter $r$

To determine the Van der Waals force an appreciation of the Hamaker constant [9] for the interacting surfaces must be gained. There are multiple methods of calculating the Hamaker constant all of which require empirical data. Additionally, Van der Waals forces can be significantly altered by surface roughness. An approximation of the Van der Waals force that includes the effects of surface roughness is given by

$$
F_{3}=\left(\frac{2 z}{2 z+r}\right)^{2} \frac{H R}{6 z^{2}}
$$

where $r$ is a constant describing the average surface roughness of the two surfaces and $H$ is the Hamaker constant for the materials involved [10]. 


\subsection{The Casimir Force}

A prediction of quantum electrodynamics is that the exclusion of electromagnetic modes will cause two closely spaced conducting plates to be mutually attracted at the micro-scale [11]. Even though this attraction is extremely weak, especially between a sphere and a plate (as opposed to two parallel plates), when the separation distance is below $1 \mu \mathrm{m}$, the Casimir force tends to be stronger than the Van der Waals force and the electrostatic force. The equation to describe the Casimir force between a sphere and a plane is

$$
F_{4}=\frac{R \pi^{3} \hbar c}{360 z^{3}}
$$

where $\hbar$ is Planck's constant divided by $2 \pi$ and $c$ is the speed of light in a vacuum.

\subsection{The Squeeze-Film Effect}

Any micro device not enclosed within a vacuum will experience damping due to the finite viscosity of air [12]. This effect is non-linear and is assumed to be negligible in the current model, but its effects will be included in any future models.

\section{Surface Interaction Model}

The micro-CMM probe was designed such that its amplitude actively counteracts the influence of any forces it is subject to while interacting with a measurement surface. These surface forces, as described in the previous section, are dependant on certain physical attributes of the system. These attributes include (but are not limited to) the densities of the two interacting materials (the probe material and the measurement surface material), the Hamaker constant for the two interacting materials and air, the depth of the combined surface imperfections of the two interacting materials, and the contact angle of any liquid contamination between the two interacting surfaces.

To simplify the model it was assumed that the materials used for interaction experiments would be similar - tungsten carbide in this case. Table 1 shows the assumed values of the physical constants used to populate the surface force and vibration model.

Table 1. Physical attributes of the probe system used in the probe-surface interaction model

\begin{tabular}{ll}
\hline Physical Attribute & Value \\
\hline Density of tungsten carbide & $15000 \mathrm{~kg} \cdot \mathrm{m}^{-3}[13]$ \\
Radius of probe tip $(R)$ & $50 \mu \mathrm{m}$ \\
Combined depth of surface imperfections $(r)$ & $100 \mathrm{~nm}$ \\
Contact angle of water with tungsten carbide $(\theta)$ & $85^{\circ}$ \\
Surface tension of water $(\gamma)$ & $0.07275 \mathrm{~J} \cdot \mathrm{m}^{-2}[13]$ \\
Hamaker constant for tungsten carbide and air $(H)$ & $21 \times 10^{-21} \mathrm{~J}[9]$ \\
Natural frequency of probe & $1.6 \mathrm{kHz}$ \\
\hline
\end{tabular}


The contact angle of water with tungsten carbide was measured using a Krüss DSA100M picolitre dosing system. The reported angle is an average value over several tungsten carbide gauge block surface samples. The depth of the surface imperfections is estimated as a worst-case value for a grade $\mathrm{K}$ tungsten carbide gauge block surface.

The analytical model has been constructed accounting for the four main surface interaction forces. In order to model the worst-case scenario, gravity was also included although it is generally regarded as insignificant at this scale. To approximate the total force that a probe would experience, the arithmetic sum of the individual surface forces was calculated. This force approximates the worst-case scenario in which all of the forces are acting against the probe and it is vibrating in the vertical direction. The minimum required amplitude of oscillation for the probe based on surface conditions, probe mass and oscillation frequency is

$$
A_{\text {min }}=\frac{2 F_{s}}{k}
$$

where $F_{s}$ is the sum of all the constituent surface forces. The strength of the individual forces, $F_{1}, F_{2}, F_{3}$ and $F_{4}$, and the total surface interaction force, $F_{s}$, are shown in Fig. 4.

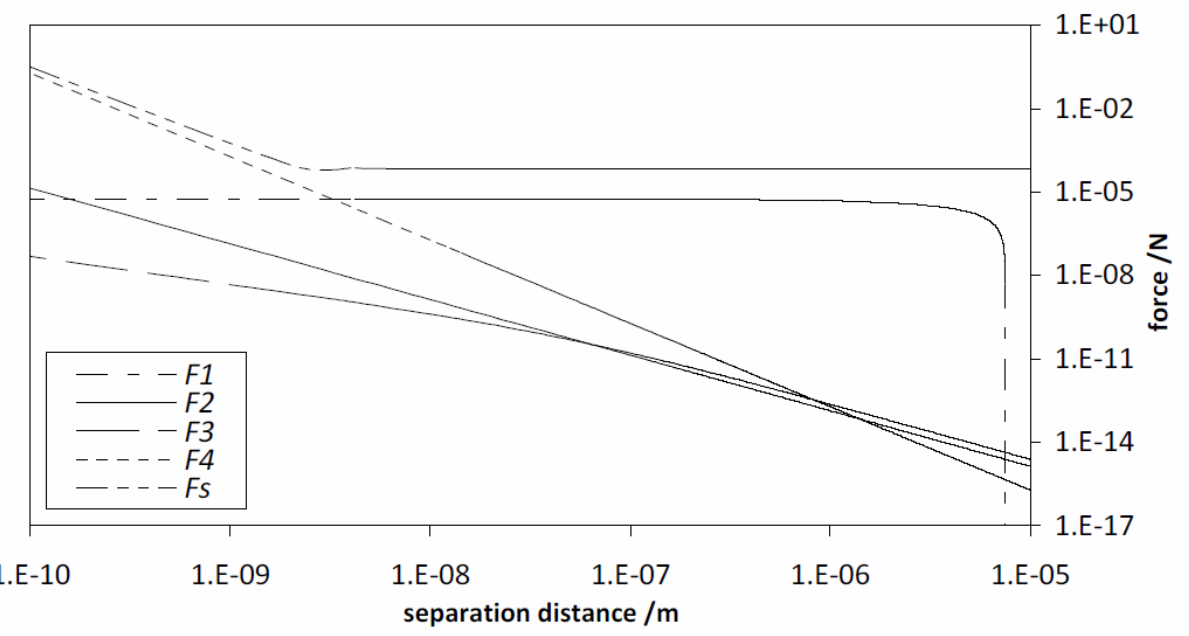

Fig. 4. Theoretical surface force strength with respect to separation distance, $z$

It can be seen that $F_{l}$ (the capillary force) dominates over a large range of separation distances. $F_{s}$, the combined surface force, includes the gravitational force on the finite mass of the device and is therefore an order of magnitude larger than $F_{l}$ at large separation distances.

By combining equations 2, 3, 4 and 5 to evaluate the total surface interaction force with respect to $z$ (the separation distance between the probe and the surface), the 
minimum amplitude required to counteract the surface forces when probing $2 \mathrm{~nm}$ from the surface was found from equation 6 to be approximately $700 \mathrm{~nm}$.

\section{Results}

Initial work on a micro-CMM probe remote from any surface investigated the amplitude of the vibration with respect to varying actuation voltages and offsets. The amplitudes were measured using a laser Doppler vibrometer. These results are shown in Fig. 5.

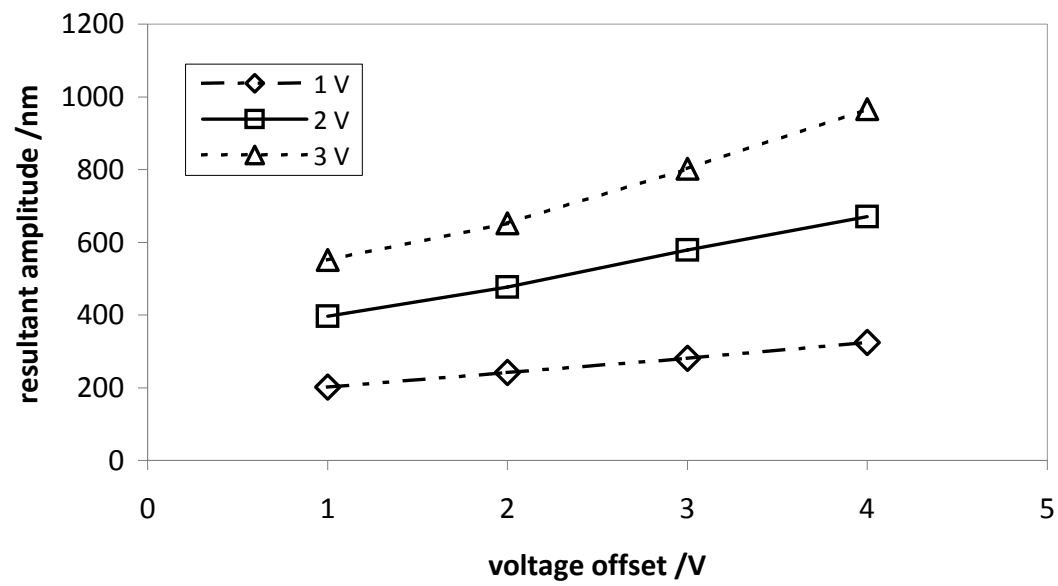

Fig. 5. Measured micro-CMM probe vibration amplitude with respect to voltage offset on the actuators for three different actuation voltage amplitudes

It can be seen from Fig. 5 that a wide range of amplitudes ranging from $200 \mathrm{~nm}$ to $1 \mu \mathrm{m}$ can be achieved with the vibrating micro-CMM probe. These experimental results, when compared to the theoretical minimum required amplitudes (as described in equation 6) suggest that the probe has suitable versatility to counteract any surface force interactions during probing.

\section{Conclusions}

In order to understand the interactions between a vibrating micro-CMM probe and a measurement surface, a model to describe a driven oscillator subject to a damping force was developed. The surface forces that constitute the damping force were investigated and modelled and, from this, an expression for the minimum required amplitude of vibration was developed. This theoretical minimum was compared to experimental data collected using a laser Doppler vibrometer, which confirmed that the vibration of the probe is suitable to counteract the surface interaction forces. 


\section{Future Work}

Work will continue on the development of the micro-CMM probe by comparing the theoretical amplitude and frequency shifts, calculated from equation 1, to real data collected from interaction experiments of the vibrating micro-CMM probe with a measurement surface.

\section{Acknowledgments}

This work was funded by the UK National Measurement Office Engineering Measurement Programme 2008 to 2011.

\section{References}

1. Leach, R.K.: Fundamental principles of engineering nanometrology. Elsevier, Amsterdam (2009)

2. Lewis, A.J.: A fully traceable miniature CMM with sub-micrometre uncertainty. In: Proc. SPIE, vol. 5190, pp. 265-276 (2003)

3. Weckenmann, A., Estler, T., Peggs, G., McMurty, D.: Probing systems in dimensional metrology. Ann. CIRP 54, 657-684 (2004)

4. Stoyanov, S., Bailey, C., Leach, R.K., Hughes, B., Wilson, A., O’Neill, W., Dorey, R.A., Shaw, C., Underhill, D., Almond, H.J.: Modelling and prototyping the conceptual design of 3D CMM micro-probe. In: Proc. 2nd ESITC, Greenwich, September 1-4, pp. 193-198 (2008)

5. Seugling, R.M., Darnell, I.M., Florando, J.N., Woody, S.C., Bauza, M., Smith, S.T.: Investigating scaling limits of a fibre based resonant probe for metrology applications. In: Proc. 12th ICPE, Portland, USA, October 19-24 (2008)

6. Lambert, P.: Capillary forces in microassembly. Springer, New York (2007)

7. Van Brussel, H., Peirs, J., Reynaerts, D., Delchambre, A., Reinhart, G., Roth, N., Weck, M., Zussman, E.: Assembly of microsystems. Ann. CIRP 49(2), 451-472 (2000)

8. Sitti, M., Hashimoto, H.: Force controlled pushing of nanoparticles: modelling and experiments. In: Proc. IEEE/ASME International Conference on Advanced Intelligent Mechatronics, Atlanta, September 19-23, pp. 13-20 (1999)

9. Andersson, K.M.: Aqueous processing of WC-Co powders. Doctoral Thesis, Royal Institute of Technology, Stockholm (2004)

10. Arai, F., Andou, D., Fukuda, T.: Adhesion forces reduction for micro manipulation based on microphysics. In: Proc. IEEE 9th Annual International Workshop on MEMS, San Diego, Febryary 11-15, pp. 354-359 (1996)

11. Lamoreaux, S.K.: Demonstration of the Casimir force in the 0.6 to $6 \mu \mathrm{m}$ range. Phys. Rev. Lett. 78(1), 5-8 (1997)

12. Sattler, R., Wachutka, G.: Compact models for squeeze-film damping in the slip flow regime. NSTI-Nanotech. 2, 243-246 (2004)

13. Kaye, G.W., Laby, T.H.: Tables of physical and chemical constants, 16th edn. Longman, London (1995), http: / /www. kayelaby.npl.co.uk/ (2008) 